Journal of the Magnetics Society of Japan Vol. 15 Supplement, No. S2 (1991)

(C) 1991 by The Magnetics Society of Japan

\title{
MAGNETIC PROPERTIES DEPENDENCE OF CO-Cr FILMS ON MAGNETRON SPUTTERING CATHODE
}

\author{
Reiji NISHIKAWA \\ Toshiba Corporation, R\&D Center \\ 1 komukai Toshiba-cho, Saiwai-ku, Kawasaki 210, Japan
}

\begin{abstract}
In order to clarify the influence of the magnetic field distribution on the magnetic properties of $\mathrm{Co}-\mathrm{Cr}$ films in a planar magnetron sputtering system, different kinds of sputtering cathodes were prepared. The point at which $\mathrm{Hc}$ for the $\mathrm{Co}-\mathrm{Cr}$ films became a maximum coincided qualitatively with the point at which the perpendicular component of the magnetic field was zero. A superimposition of the magnetic field normal to the target can be expected to induce a shift of this point, and a change in magnetic properties. In roll sputtering, the caracteristics of the cathode affected the magnetic properties of the $\mathrm{Co}-\mathrm{Cr}$ film at lower roll temperatures, but at higher roll temperatures, the magnetic properties of the Co-Cr films were little influenced by the distribution of plasma density.
\end{abstract}

\section{Introduction}

The technology of dc planar magnetron sputtering is suitable for the production of $\mathrm{Co}-\mathrm{Cr}$ perpendicular magnetic recording media[1]. It is well known that the magnetic field plays an important role in the planar magnetron sputtering system. The erosion pattern of the sputtering target depends on the magnetic field, and this strongly influences the columnnar structure and the direction of easy magnetization[2][3] .

Superimposition of the magnetic field normal to the target is known to affect the magnetic properties of $\mathrm{Co}-\mathrm{Cr}$ films, especially coercive force $\mathrm{Hc}$ [4]. However, the relationship between the magnetic field distribution in the plasma discharge region and the magnetic properties of the Co-Cr film has not been clarified.

In this paper, the relationship mentioned above is described. The relationship between effective temperature of the substrate during deposition and $\mathrm{Hc}$ of the Co-Cr film will be also discussed.

\section{Experimental}

Different types of planar magnetron sputtering cathodes and sputter apparatus were used. The magnetic circuits of the cathodes were constructed with rare earth cobalt permanent magnets and iron yokes.
The $X, Y$, and $Z$ components of magnetic field were measured using a Hall-type gaussmeter Magnetic properties of the Co-Cr films were measured with a vibrating sample magnetometer.

Temperature of the substrate films was measured using a $0.1 \mathrm{~mm}$-diameter alumelchromel thermocouple glued to the substrate. The thermocouple in the vacuum chamber was completely covered with adfesives or a teflon tube to prevent the inflow of electrons or argon ions from the plasma discharge region. Polyimid films were used for the substrates.

\section{Results}

Two types of planar magnetron sputtering cathodes, cathode $A$ and cathode $B$, whose magnetic circuits were different, were used in the study. The influence of the magnetic field distribution on the sputtering characteristics and the magnetic properties of the $\mathrm{Co}-\mathrm{Cr}$ films was measured. The $\mathrm{Ar}$ gas pressure during sputtering was $0.65 \mathrm{~Pa}$. Before deposition, the chamber was pumped down to less than $10^{-4} \mathrm{~Pa}$ using an oil diffusion pump. The separation between substrate and target was $100 \mathrm{~mm}$, and the window length was $50 \mathrm{~mm} .25 \mu \mathrm{m}$-thick polyimid film was moved on a water cooled holder.

Figure 1 shows the dependence of $\mathrm{Hc}$ on sputtering power for the $\mathrm{Co}-\mathrm{Cr}$ films. The 


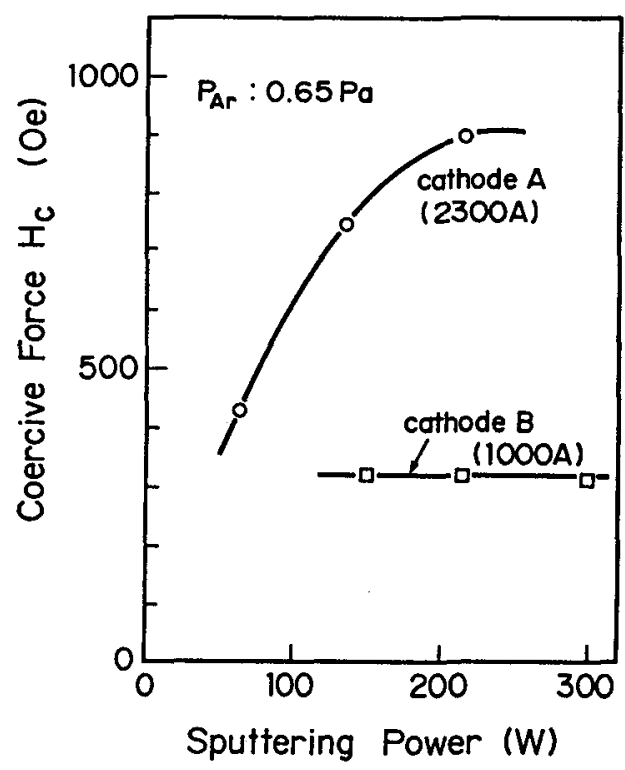

Fig. 1 Dependence of $\mathrm{Hc}$ on sputtering power for $\mathrm{Co}-\mathrm{Cr}$ films deposited by cathode $A$ and cathode $B$

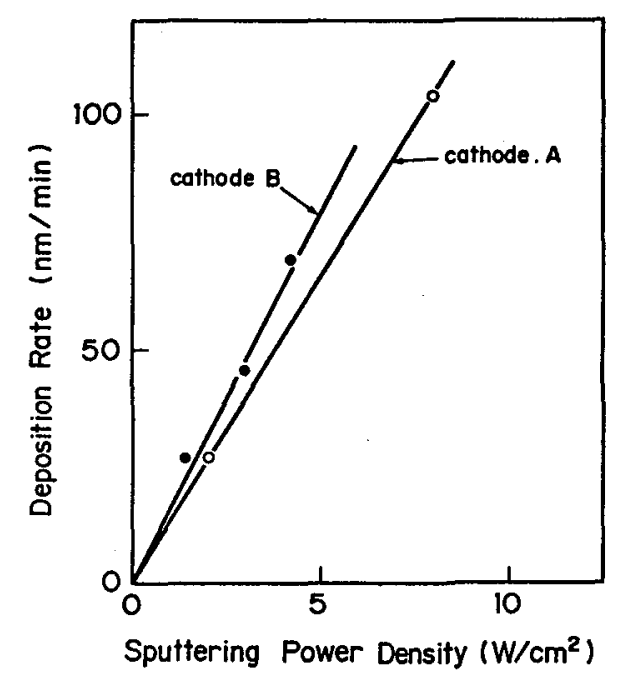

Fig. 2 Deposition rate dependence on sputtering power density for cathode A and cathode B

Hc value for films deposited by the cathode $A$ was higher and depended on the sputtering power. However, Hc for films deposited by cathode $B$ was small and did not show sputtering power dependence. The power dependence of the deposition rate for both cathodes is almost the same (Fig.2). I-V characteristics for both cathodes are shown in Fig. 3. Though the cathode voltage for cathode B was high in the low-power region, as sputtering power increased, the voltage became lower than that for cathode $\mathrm{A}$.

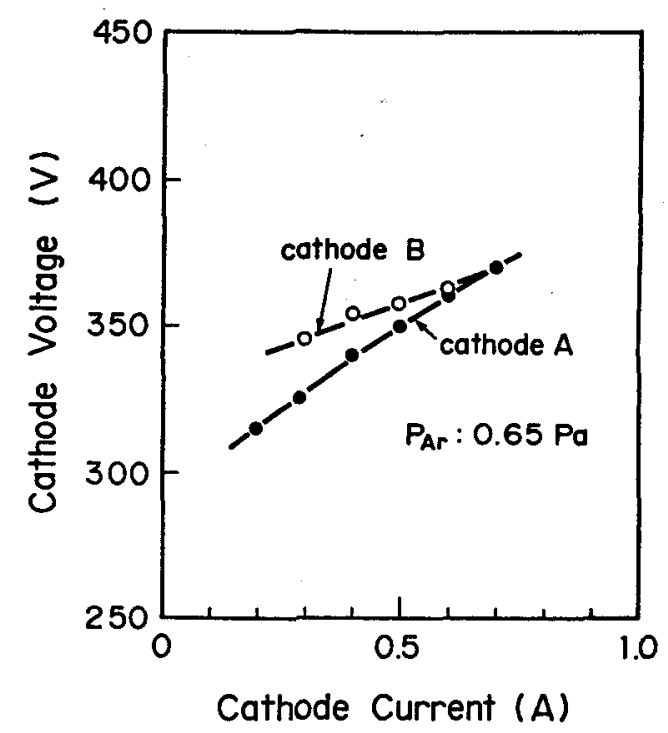

Fig. 3 I-V characterstics for cathode A and cathode B

The magnetic field parallel to the target is well-known to strongly affect the discharge characteristics during magnetron sputtering. The value of the magnetic field parallel to the target for both cathodes differed little in 0 to 7.5 $\mathrm{mm}$ height from the target level(Fig.4). This result suggests that the magnetic properties of the Co-Cr films, and especially $\mathrm{Hc}$, are affected not only by the magnetic field strength parallel to the target surface but by the distribution of the magnetic field.

Figures $5 \mathrm{a}$ and $5 \mathrm{~b}$ show schematics of the magnetic field distribution for cathode $A$ and cathode $B$. These magnetic fields are very different.

Figure 6 shows the Hc distribution of a 50nm-thick Co-Cr film deposited onto a stationary substrate by another rectangular cathode with a $120 \mathrm{~mm} \times 250 \mathrm{~mm}$ target (cathode $\mathrm{C}$ ). The magnetic circuit of cathode $C$ is similar to that of cathode $A$. 


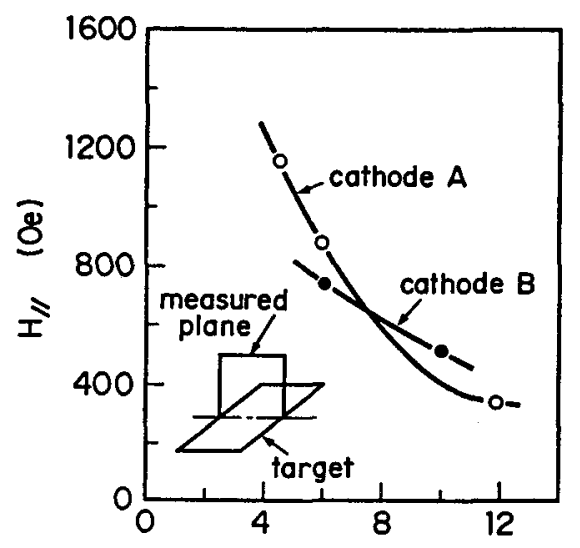

Distance from target surface $(\mathrm{mm})$

Fig. 4 Intensity of magnetic field for cathode A and cathode B

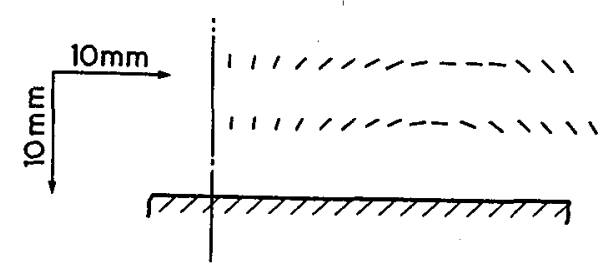

Fig.5b Magnetic field distribution for cathode $B$

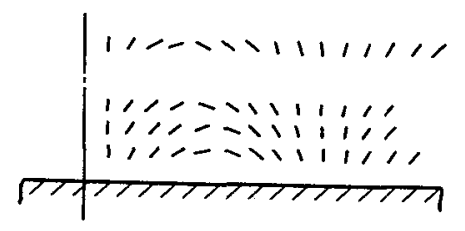

Fig.5a. Magnetic field distribution for cathode A

Figure 7 shows the magnetic field distribution in the plane normal to the target and on the longitudinal center axis. The point at which the perpendicular component of the magnetic field becomes zero shifted from outside to inside of the space between target and substrate as distance from the target increased.

This phenomenon is due to magnetic saturation of the inner magnets of the racetrack shaped magnetic circuit.
It is known that at the point where the magnetic field is parallel to the target, the plasma is dense and the absolute value of the floating potential is higher relative to other positions.

The relatively dense plasma is thought to induce an increase of substrate temperature. Therefore, the Hc distribution described above can be explained by the plasma behavier resulting from the magnetic field distribution.

On the other hand, the point at which the perpendicular component of the magnetic field becomes zero for cathode B shifted outside as distance from the target increased. Therefore, a substrate is thought to be exposed little to dense plasma. These results mean that the $\mathrm{Hc}$ of the magnetron sputtered $\mathrm{Co}-\mathrm{Cr}$ films and the plasma behavier can be controlled by changing of the magnetic field distribution.

It can be seen in Fig. 7 that in a plane above some critical height, there is no point at which the perpendicular component of the magnetic field becomes zero on the target. This type of the magnetron sputter ing cathode engages to obtain high HC Co-Cr films.

A superimposition of the magnetic field normal to the planar magnetron sputtering cathode induces a shift in the position at which the perpendicular component of the magnetic field becomes zero. In a case where the superimposed magnetic field is larger than some critical strength, the mag netic field distribution becomes similar to that for the specially designed cathode described above.

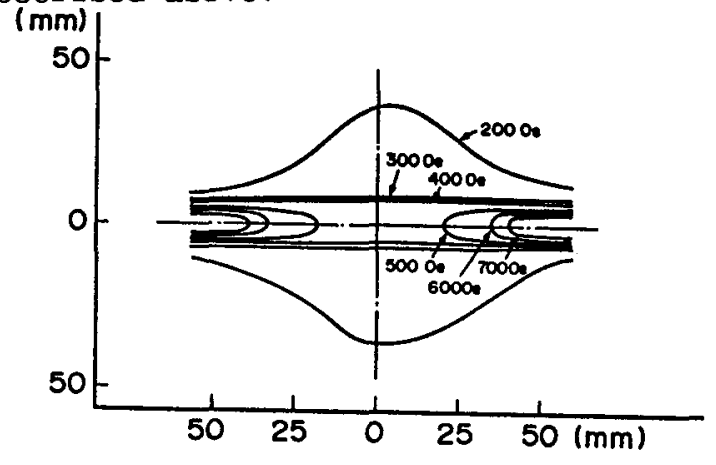

Fig. $6 \mathrm{Hc}$ distribution for $\mathrm{Co}-\mathrm{Cr}$ film sputtered on stationary substrate cathode $\mathrm{C}$ 


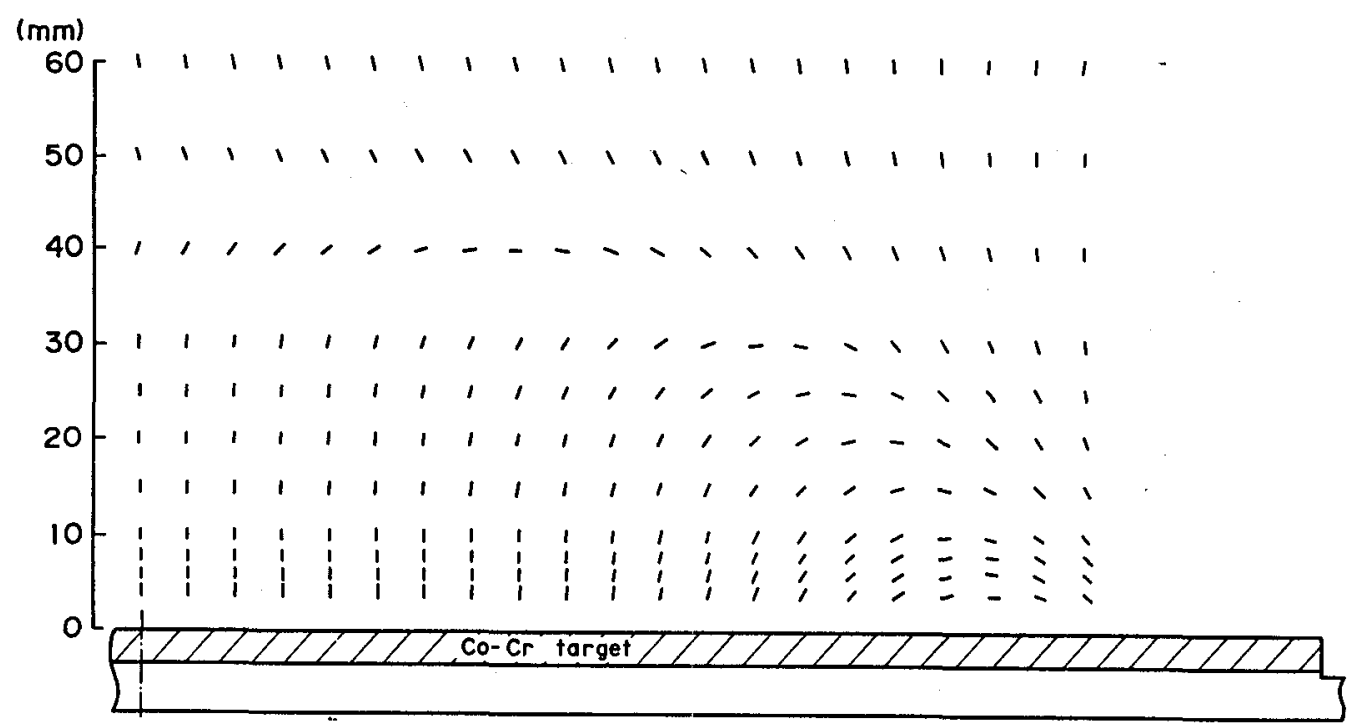

Fig. 7 Magnetic field distribution for cathode $\mathrm{C}$ in plane normal to target and on longitudinal center axis

Figure 8 shows the relationship between the substrate temperature, Hc and the substrate holder potential for Co-Cr films deposited under control of the discharge condition with grid current control. Irrespective of the discharge condition,

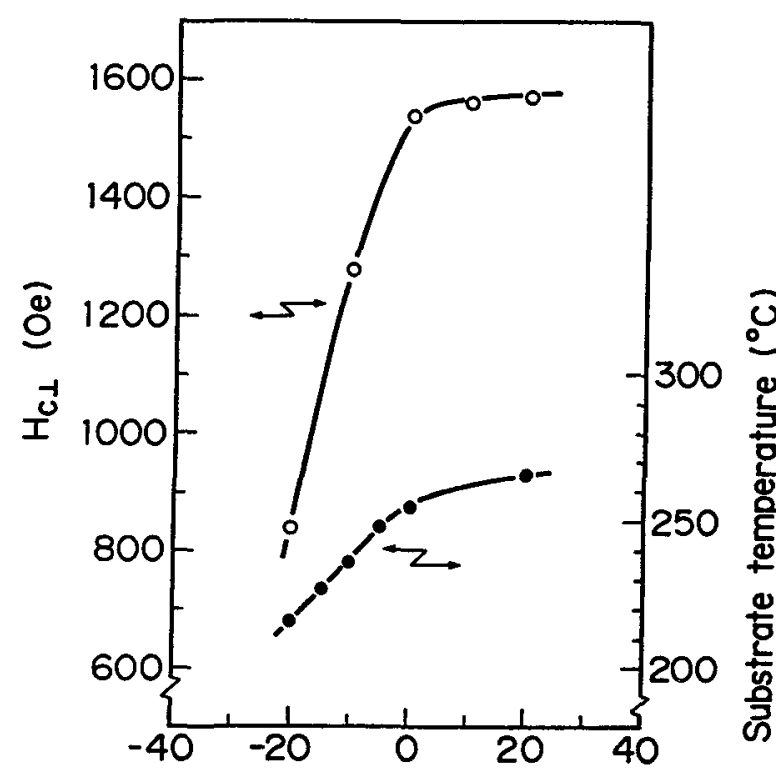

Potentiol of substrate holder (V)

Fig. $8 \mathrm{Hc}_{\perp}$ and substrate temperature dependence on potential of substrate holder the Hc value can be described principally by the surface temperature. It is clear that an effective temperature is the most important function for a determination of the $\mathrm{Hc}$ of $\mathrm{Co}-\mathrm{Cr}$ films.

To apply magnetron sputtering cathodes to roll sputtering, the thermal flow between the roll and the substrate film must be taken into consideration.

Figure 9 shows the Hc variation over the film length for roll-sputtered $\mathrm{Co}-\mathrm{Cr}$ films at $150^{\circ} \mathrm{C}$. In the early stages of sputtering. Hc values change irregularly, but with a tendency to increase gradually. It finally reached a constant value determined by the roll temperature. The variation in film curl, which is indicative of internal stress, is similar to the variation in Hc. This means the variation in $\mathrm{Hc}$ is due to the variation in thermal flow from the substrate to the roll, because the substrate temperature is determined by the roll temperature when the substrate is not exposed to plasma.

These results suggest that in the case of relatively low roll temperature and locally dense plasma, a distribution in Hc for $\mathrm{Co}-\mathrm{Cr}$ films will be induced. 


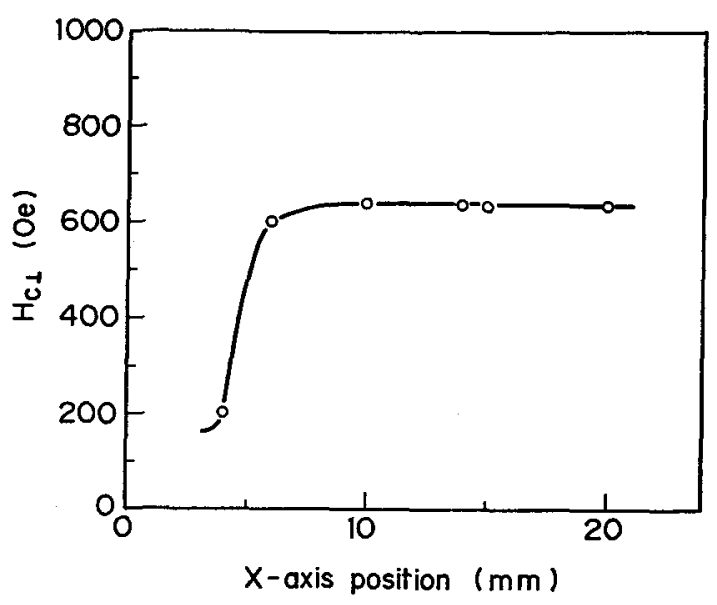

Fig. $9 \mathrm{Hc}_{\perp}$ variations along substrate moving direction

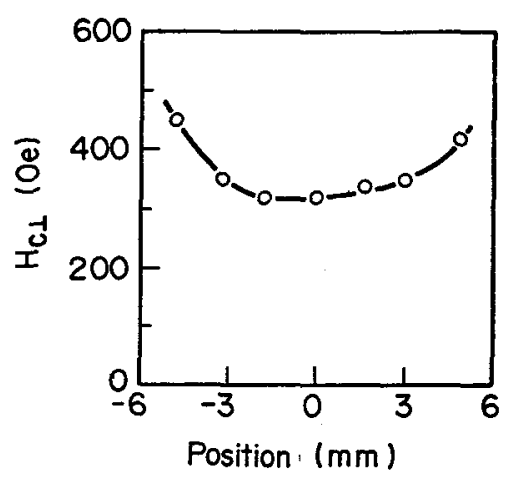

Fig. 10 Hc variation along transverse direction

\section{Conclusion}

In the magnetron sputtering of the CoCr films, plasma density, as determined by the magnetic field distribution, plays an important role in the determination of the Hc value. Superimposition of a normal field to a planar magnetron sputtering cathode, induces a shift of the point where the plasma density is a maximum and the Hc value reaches a maximum in a plane parallel the target. In roll sputtering, the thermal flow from substrate to roll influenced the magnetic properties of the Co-Cr films. At low roll temperature, plasma behavier affected the Hc of the magnetron sputtered Co-Cr films.

Acknow ledgement

The author thank Mr.Satoyama for his discussion and guidance.

\section{References}

[1]R.Nishikawa, T.Suzuki, and T.Sonoda, Toshiba Review, No.154,32(1985)

[2]Y.Tanaka, H. I to, T.Sonoda and R. Nishikawa IEEE Trans. Magn., MAG-23, No. 5, 2046 (1987)

[3]N. Inoue and R.Nishikawa, J.of the Mag. Soc. Japan, 13, supple. , No. $\$ 1,703$ (1989)

[4]Y.Uchiyama, H.Sato, Y.Kitamoto and U.Hwang, IEEE Trans. Magn. ,MAG-26, No.5, 1617 (1990)
Figure 10 shows variation in $\mathrm{Hc}$ measured along transverse direction when sputtered with cathode $\mathrm{C}$ at $90^{\circ} \mathrm{C}$. As reflection of the variation of the plasma density, the variation in $\mathrm{Hc}$ along the transverse direction was observed. This reslut indicates that the $\mathrm{Hc}$ of the roll sputtered Co-Cr film is affected not only by the roll temperature, but by the plasma behavier of the magnetron sputtering. A well designed magnetron sputtering cathode made possible to produce high $\mathrm{Hc} \mathrm{Co}-\mathrm{Cr}$ films uniformly. 